\title{
On the Origin of Iron/Sulfur Cluster Biosynthesis in Eukaryotes
}

\author{
Anastasios D. Tsaousis* \\ Laboratory of Molecular and Evolutionary Parasitology, ResistAnce Pathogenicity and Infectious Diseases (RAPID) Group, \\ School of Biosciences, University of Kent, Canterbury, United Kingdom
}

Iron and sulfur are indispensable elements of every living cell, but on their own these elements are toxic and require dedicated machineries for the formation of iron/sulfur (Fe/S) clusters. In eukaryotes, proteins requiring Fe/S clusters (Fe/S proteins) are found in or associated with various organelles including the mitochondrion, endoplasmic reticulum, cytosol, and the nucleus. These proteins are involved in several pathways indispensable for the viability of each living cell including DNA maintenance, protein translation and metabolic pathways. Thus, the formation of Fe/S clusters and their delivery to these proteins has a fundamental role in the functions and the evolution of the eukaryotic cell.

OPEN ACCESS

Edited by:

Baolei Jia,

Chung-Ang University, South Korea

Reviewed by:

Saman Habib,

Central Drug Research Institute (CSIR), India

Annalisa Pastore,

King's College London, United Kingdom John Markley,

University of Wisconsin-Madison, United States

*Correspondence:

Anastasios D. Tsaousis

a.tsaousis@kent.ac.uk;

tsaousis.anastasios@gmail.com

Specialty section:

This article was submitted to

Evolutionary and Genomic

Microbiology,

a section of the journal

Frontiers in Microbiology

Received: 04 July 2019

Accepted: 15 October 2019

Published: 08 November 2019

Citation:

Tsaousis AD (2019) On the Origin of Iron/Sulfur Cluster Biosynthesis in Eukaryotes.

Front. Microbiol. 10:2478. doi: 10.3389/fmicb.2019.02478
Currently, most eukaryotes harbor two (located in cytosol and mitochondrion) or three (located in plastid) machineries for the assembly of Fe/S clusters, but certain anaerobic microbial eukaryotes contain sulfur mobilization (SUF) machineries that were previously thought to be present only in archaeal linages. These machineries could not only stipulate which pathway was present in the last eukaryotic common ancestor (LECA), but they could also provide clues regarding presence of an Fe/S cluster machinery in the protoeukaryote and evolution of $\mathrm{Fe} / \mathrm{S}$ cluster assembly machineries in all eukaryotes.

Keywords: last eukaryotic common ancestor, iron sulfur cluster biogenesis, sulfur mobilization machinery, iron sulfur cluster machinery, eukaryotic evolution, cytosolic iron/suphur cluster assembly machinery

\section{INTRODUCTION}

Iron/sulfur (Fe/S) clusters are fundamental and ubiquitous factors. All living cells have biosynthetic machineries responsible for their assembly and delivery, since the individual components [iron $(\mathrm{Fe})$ and sulfur (S)] are toxic for the cells themselves (Lill et al., 1999; Lill, 2009). Importantly, $\mathrm{Fe} / \mathrm{S}$ clusters are essential factors of proteins involved in essential functions of the cell including, but not restricted to, photosynthesis, respiration, DNA replication and repair, and regulation of gene expression (Lill et al., 2012). Eukaryotes are not the exception to this paradigm. The typical Fe/S biosynthetic machineries found in bacteria and archaea have also been identified in eukaryotes, but compartmentalization and evolution of these machineries in several eukaryotes are still under investigation. A typical eukaryotic cell harbors the iron-sulfur cluster (ISC) in the mitochondria and the cytosolic iron/suphur cluster assembly (CIA) machinery in the cytosol, while plastid-carrying cells also harbor the sulfur mobilization (SUF) machinery in their plastids.

Among those, the ISC machinery has been considered to be the reason for the existence of mitochondria (Lill et al., 1999; Lill, 2009; Hjort et al., 2010) and fundamental for the evolution of eukaryotes. Nonetheless, what happens when a eukaryote does not harbor any mitochondria (Karnkowska et al., 2016)? Could this organism provide some clues about the presence of $\mathrm{Fe} / \mathrm{S}$ biosynthetic machineries in the early eukaryotes and their role in the evolution of the eukaryotic cell? 


\section{IRON-SULFUR CLUSTER ASSEMBLY IN MITOCHONDRIAL DIVERSITY}

It is widely accepted that mitochondria originated from or within the alpha-proteobacteria (Gray et al., 1999, 2001; Gawryluk, 2018; Martijn et al., 2018), whereby the latter was "engulfed" by a eukaryotic host and potentially gave rise to the last eukaryotic common ancestor (LECA). Nevertheless, questions regarding why, how, and when this event took place are still under debate (Gray et al., 2001; Embley and Martin, 2006; Lane and Martin, 2015, 2016; Martin et al., 2016; Pittis and Gabaldon, 2016; Gabaldon, 2018). It is quite apparent from accumulated data that the acquisition of mitochondria has been the decisive step in eukaryogenesis (Martin et al., 2016). One hypothesis postulates that the mitochondria fulfilled energy requirements of the cell thus their presence provided a selective advantage to the organisms bearing them to become eukaryotes (Lane and Martin, 2015, 2016; Pittis and Gabaldon, 2016). Another hypothesis, which does not exclude others, suggests that the reason for the existence of mitochondria could have been the assembly of Fe/S clusters (Lill et al., 1999), the latter being the only mitochondrial biosynthetic pathway that is essential for survival of eukaryotic cells. So far, this has been shown experimentally in yeast (Braymer and Lill, 2017), mammalian cells (Rouault and Maio, 2017), and trypanosomes (Pena-Diaz and Lukes, 2018).

Further support to this hypothesis arose from investigations in previously considered "primitive" amitochondriate eukaryotes. These organisms were shown to harbor mitochondrial-related organelles (MROs), a secondarily reduced form of mitochondria, including hydrogen producing organelles called hydrogenosomes in Trichomonas (Muller, 1973), or highly reduced remnant organelles called mitosomes, which were found in Giardia (Tovar et al., 2003); Microsporidia (Williams et al., 2002; Tsaousis et al., 2008), and Entamoeba (Tovar et al., 1999). Whether a "primitive" amitochondriate eukaryote could exist or not is still under debate (Margulis et al., 2006). Nonetheless, a eukaryote that secondarily lost its mitochondria was identified recently (Karnkowska et al., 2016). Interestingly, the only biosynthetic pathway conserved in all these organelles is the assembly of $\mathrm{Fe} / \mathrm{S}$ clusters, providing further support on the necessity/ importance of this machinery for cell viability. From an evolutionary standpoint, it will be important to elucidate how the eukaryotic cell supported its needs for Fe/S clusters, before the acquisition of mitochondria. To provide insight on this matter, I will first need to examine the distribution of various $\mathrm{Fe} / \mathrm{S}$ cluster machineries in eukaryotic cells and their necessity to the host's functions, followed by various theories on the evolution of Fe-S cluster machineries across eukaryotes.

\section{MITOCHONDRIAL IRON/SULFUR CLUSTER MACHINERY}

All mitochondria investigated so far possess some semblance of an $\mathrm{Fe} / \mathrm{S}$ cluster biosynthetic pathway for de novo assembly of $\mathrm{Fe} / \mathrm{S}$ clusters into organellar apo-proteins (see below) but potentially for the support of cytosolic and nuclear apo-proteins as well (Lill, 2009; Ali and Nozaki, 2013). The typical mitochondrial machinery is the iron-sulfur cluster (ISC), which is comprised of 18 (currently known in yeast) proteins (Braymer and Lill, 2017), all of which are involved in the biogenesis and trafficking of clusters in mitochondria (Figure 1). The process is divided into four stages (for detailed review, see Braymer and Lill, 2017): (1) de novo [2Fe-2S] cluster synthesis; (2) trafficking of [2Fe-2S] clusters and insertion into mitochondrial apo-proteins, or mitochondrial export of an as yet unknown sulfur-containing species (X-S) to the cytosol; (3) conversion of [2Fe-2S] into [4Fe-4S] clusters; and lastly (4) trafficking of [4Fe-4S] clusters and insertion into mitochondrial [4Fe-4S] apo-proteins (e.g., lipoate synthase, succinate dehydrogenase, and components of respiratory complex I). Most organisms harboring mitochondria encode some of these components, including organisms with remnant mitochondria such as Giardia (Tovar et al., 2003), Cryptosporidium (Miller et al., 2018), and Microsporidia (Goldberg et al., 2008; Freibert et al., 2017), in which ISC stages 3 and 4 are lacking ([4Fe-4S] cluster synthesis and targeting; Figure 1), due to the lack of mitochondrial apo-proteins requiring [4Fe-4S] clusters.

\section{CYTOSOLIC IRON/SULFUR CLUSTER MACHINERY}

All eukaryotes require a cytosolic Fe/S cluster (CIA) machinery to support cytosolic and nuclear Fe/S cluster proteins (Tsaousis et al., 2014). So far, 11 proteins have been identified in both mammals and yeast as responsible for synthesis, trafficking, and insertion of clusters in the cytosol and the nucleus (Braymer and Lill, 2017; Tonini et al., 2018). Of these, several CIA protein complexes support different stages in the process (Figure 2A). For example, a bridging [4Fe-4S] cluster is assembled on the Cfd1-Nbp35 complex, which depends on the as yet unidentified molecule X-S from the mitochondrial ISC machineries. Subsequently, the electron transfer chain from NADPH via the diflavin reductase Tah18 and the Fe/S protein Dre2 is required. In the next phase, the transiently bound [4Fe-4S] cluster of Cfd1-Nbp35 is transferred to and inserted into apo-proteins by the $\mathrm{Fe} / \mathrm{S}$ protein Narl, and the CIA targeting complex consisting of Cia1, Cia2, and Mms19 (Stehling et al., 2012, 2013). This entity also binds the Lto1-Yae1 adapter complex via a conserved C-terminal tryptophan in Lto1 to recruit the $\mathrm{ABC}$ protein Rli1 (participates in ribosome assembly and ribosome recycling) for dedicated assembly of its two [4Fe-4S] clusters (Lill et al., 2015; Paul et al., 2015). The CIA machinery may also support ATP-dependent DNA helicases such as Rad3, XPD, FANCJ, and RTEL1, which are involved in DNA damage repair and telomere maintenance (Rudolf et al., 2006). Interestingly, mitochondria or related organelles, such as hydrogenosomes and mitosomes (see above) seem to be essential for the support of the CIA machinery in the biogenesis of cytosolic and nuclear Fe/S clusters (Stehling et al., 2014; Tsaousis et al., 2014; Freibert et al., 2017). Despite this, organisms harboring these "reduced" mitochondria appear to lack certain components of the CIA machinery (e.g., Tah18, 


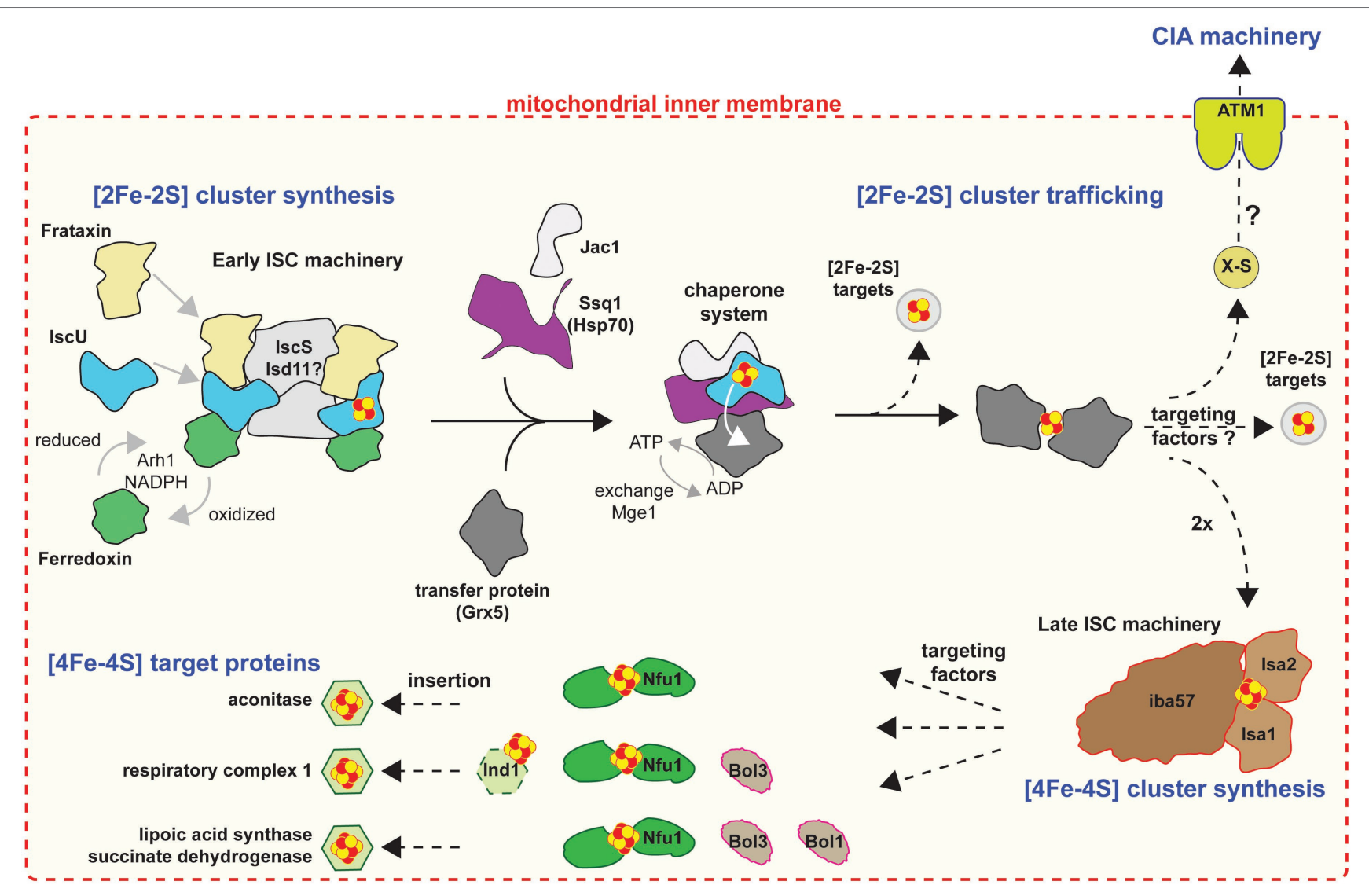

FIGURE 1 | Cartoon model of the mitochondrial Fe/S protein assembly process. Figure was produced based on Braymer and Lill (2017). A cascade of ISC proteins is required for the de novo synthesis of [2Fe-2S] and [4Fe-4S] clusters and their proper trafficking to target apoproteins in mitochondria. Initially, a [2Fe-2S] cluster is synthesized by the early ISC machinery, composed of the Isu1 scaffold protein requiring sulfide from the cysteine desulfurase complex Nfs1-Isd11-Acp1, electrons from the transfer chain NADPH-Arh1 and the ferredoxin Yah1, and the regulator and/or iron donor Yfh1. The Isu1-bound [2Fe-2S] cluster is then delivered to the monothiol glutaredoxin Grx5, a reaction accomplished by the Hsp70 chaperone Ssq1 with the help of the J-type co-chaperone Jac1. This reaction is dependent on ATP hydrolysis by Ssq1. The exchange factor Mge1 facilitates the exchange of ADP for ATP. The resulting bridging [2Fe-2S] cluster on a Grx5 dimer is inserted directly into [2Fe-2S] recipient apoproteins or trafficked to the late ISC machinery for [4Fe-4S] cluster biogenesis. The early ISC machinery, including the chaperones and Grx5, is also responsible for generating the component X-S for transport of sulfur out of the mitochondria to the CIA machinery for cytosolic-nuclear Fe/S protein biogenesis. The late ISC machinery consists of the yet structurally and functionally uncharacterized Isa1-Isa2-Iba57 complex and is needed for the generation of [4Fe-4S] clusters. Trafficking and insertion of the [4Fe-4S] clusters into target Fe/S proteins are facilitated by specific ISC targeting factors, such as Nfu1, the complex I-specific Ind1, and the Bol proteins. Dashed arrows indicate steps that remain poorly elucidated on the biochemical level.

Dre2, and Cfd1) that are otherwise essential in mammals and yeast (Tsaousis et al., 2014; Vacek et al., 2018). Even more intriguingly, microbial organisms such as cryptophytes and chlorarachniophytes that harbor cytosols from two organisms (main and cytosol of their phototrophic symbiont), seem to have two diverse and functional CIA machineries-one in each compartment-which are supported by their corresponding organelles (Grosche et al., 2018).

\section{PLASTID IRON/SULFUR CLUSTER MACHINERY}

Apo-proteins in plastids and plastid-related organelles are supported by the sulfur mobilization (SUF) machinery, which was acquired from Cyanobacteria. The six major proteins that encompass the bacterial-type SUF machinery are also present in plastids (SufA, SufB, SufC, SufD, SufE, and SufS; Figure 3A), one of which (SufC) is commonly encoded by the plastid genome (Le Corguille et al., 2009). Using genetic and biochemical investigations in prokaryotes, it was shown that SufE and SufS are involved in the sulfur mobilization from cysteine, while SufB, SufC, and SufD form a complex where SufB harbors both the de novo assembled Fe/S clusters and a flavin redox cofactor (Couturier et al., 2013). However, recent experimental structural studies have shown a dynamic motion of the $\mathrm{SufB}_{1}$ $\mathrm{SufC}_{2}-\mathrm{SufD}_{1}$ complex that could be universally applicable to all the SUF systems, including the archaeal $\mathrm{SufB}_{2}-\mathrm{SufC}_{2}$ complex (Hirabayashi et al., 2015) (discussed below). In addition, SufA could act as a carrier protein, along with numerous other carrier proteins that are currently found (Fontecave et al., 2005; Wollers et al., 2010; for review, see Couturier et al., 2013). As such, the plastidial $\mathrm{Fe} / \mathrm{S}$ assembly machinery has been mostly characterized in Arabidopsis thaliana, where 15 proteins 

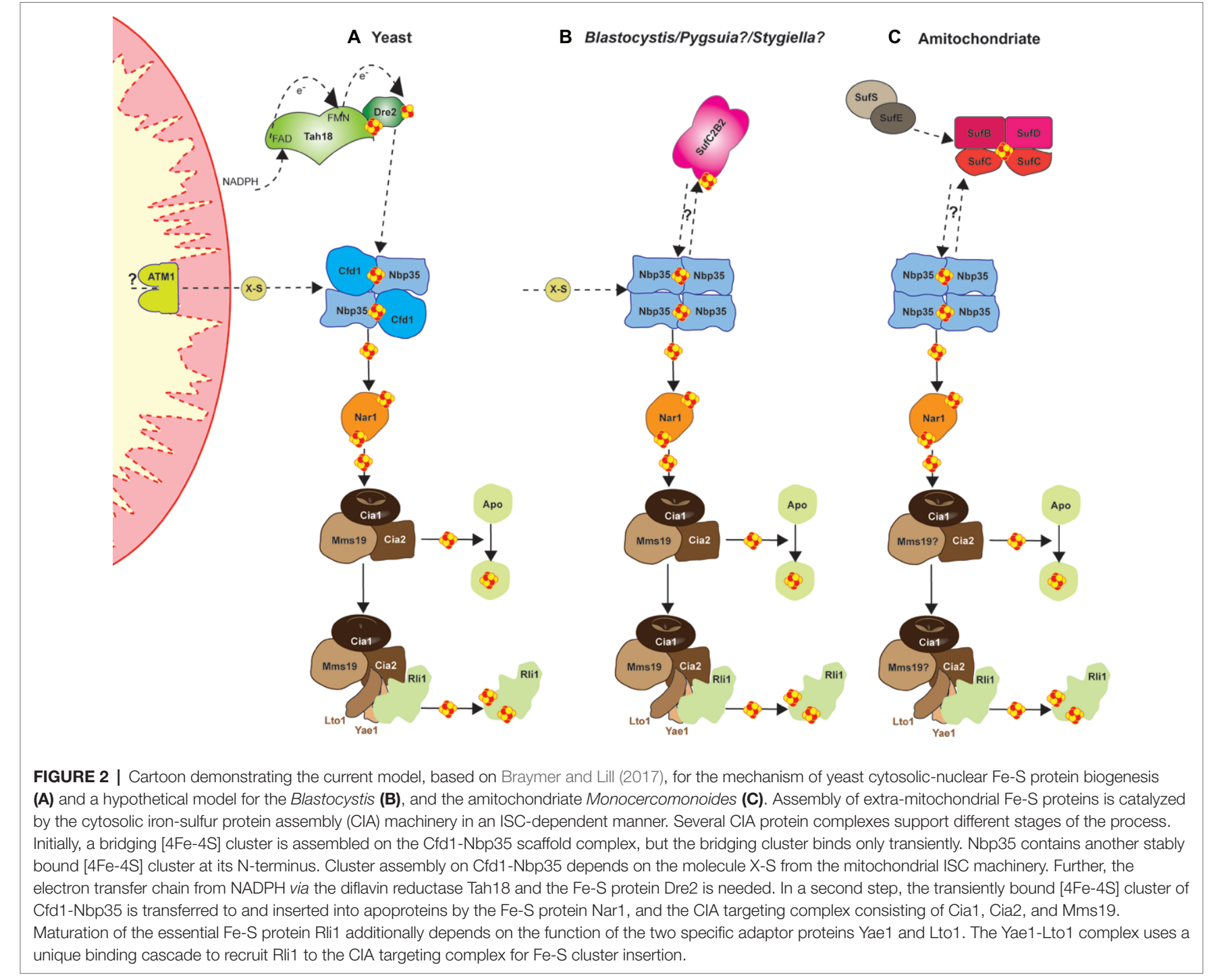

C Amitochondriate

have been experimentally localized and one of which (SufSE) was shown to be targeted in both the plastids and mitochondria (Balk and Pilon, 2011; Couturier et al., 2013). To that end, the plastidial Fe/S cluster assembly is responsible for the support of housekeeping apo-proteins of the organelle and currently is unclear if it can support the CIA machinery in cytosol of the cells (similar to the ISC machinery).

\section{IRON/SULFUR CLUSTER ASSEMBLY IN AMITOCHONDRIATES}

The discovery of a eukaryote that secondarily lost its mitochondria (Karnkowska et al., 2016) raises the question of $\mathrm{Fe} / \mathrm{S}$ cluster biosynthesis in this organism, since this is the only biosynthetic function found in all mitochondria-related organelles investigated so far (Hjort et al., 2010; Santos et al., 2018). The oxymonad Monocercomonoides sp. (currently named M. exilis; Treitli et al., 2018) is the first eukaryotic organism with no microscopic evidence for the existence of a mitochondrion. This finding was further supported by extensive genome surveys that failed to find any mitochondrial proteins, including homologues of the mitochondrial ISC pathway (Karnkowska et al., 2016). Despite this, the genome of Monocercomonoides does encode components of the CIA machinery (Figure 2C), in addition to homologs of a SUF system (Figures 3A,B). The origin of these SUF homologues though unclear seems to be bacterial (Karnkowska et al., 2016) (see below). Due to the lack of an in situ transfection system, Monocercomonoides SufC and SufB homologs were heterologously expressed in Trichomonas vaginalis and Saccharomyces cerevisiae, whereby they both localized in the cytosol of both organisms (Karnkowska et al., 2016).

Recent investigations by Vacek et al. (2018) demonstrated that oxymonads and organisms (Preaxostyla group, Metamonada, and Excavata) related to $M$. exilis also harbor an SUF machinery (Vacek et al., 2018). Genomic and transcriptomic surveys have shown the presence of components of the SUF machinery in six additional closely related species, 


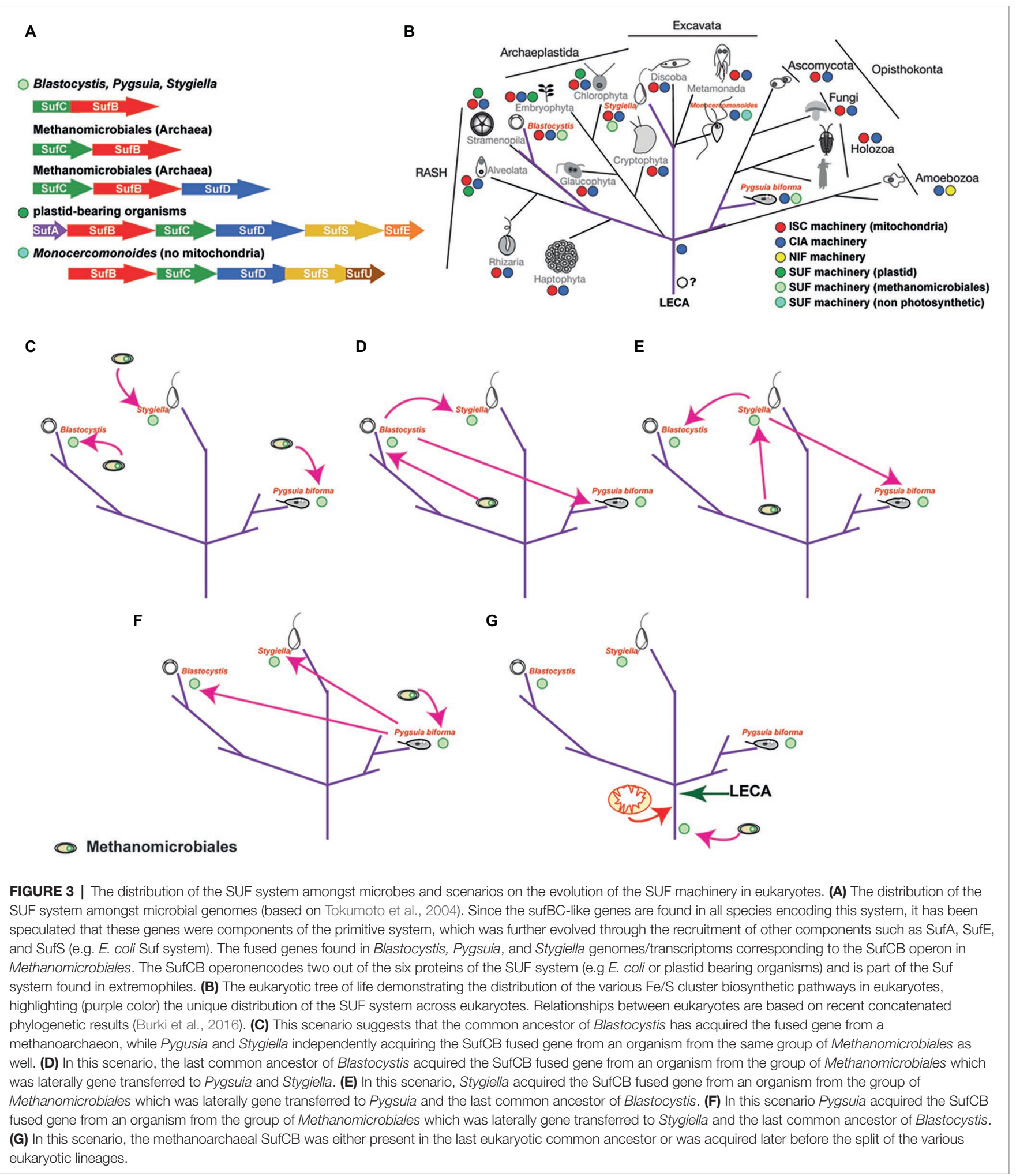

suggesting that transition from ISC to SUF preceded the last common ancestor of the lineage (Vacek et al., 2018). A follow-up inventory of all the homologues of the CIA machinery in these organisms showed that its major components are still present, consistent with previous observations that the lack of mitochondria or more specifically of the ISC machinery did not have any effect in the maturation of cytosolic $\mathrm{Fe} / \mathrm{S}$ proteins (Vacek et al., 2018). 


\section{EXCEPTIONS TO THE STATUS QUO (ALTERNATIVE DIRECTIONS)}

\section{The Case of Entamoeba and Mastigamoeba}

In addition to the machineries described above, some organisms have acquired new processes for the de novo assembly of their Fe/S clusters. The genomes of the amoebozoans Entamoeba histolytica and Mastigamoeba balamuthi (both thriving in low-oxygen environments) do not encode any components of the ISC machinery and instead they harbor a nitrogen fixation (NIF) machinery that was laterally acquired from an epsilon proteobacterion (Ali et al., 2004; van der Giezen et al., 2004). Components of the machinery were shown to localize in the mitosome of E. histolytica (Maralikova et al., 2010) (though this is still under debate; Nyvltova et al., 2013), while replica components of $M$. balamuthi were shown to localize in both the cytosol and its hydrogenosomal-like structures (Nyvltova et al., 2013). It is still unclear whether the function of a NIF system could be more advantageous over the ISC system, but it seems to be the "preferred" way in this lineage. Despite this alteration, components of the CIA machinery are present in both organisms (Tsaousis et al., 2014; Pyrih et al., 2016) (with the exception of Tah18, Dre2, and Cfd1), suggesting that ISC machinery might not (as previously thought; Lill et al., 1999) be indispensable for the function of the CIA machinery.

\section{The Case of Blastocystis, Pygsuia, Stygiella, and Others?}

Blastocystis is an obligatory anaerobic stramenopile. Blastocystis was the first non-photosynthetic eukaryotic organism to be shown to encode an ancient SUF system (Tsaousis et al., 2012), in addition to an ISC machinery that is localized in mitochondria (Tsaousis et al., 2012) and a CIA machinery that is localized in the cytosol (Tsaousis et al., 2014). The SUF system of Blastocystis is similar to the one of Methanomicrobiales in that both display fusion of the SufC and SufB genes. Phylogenetic analysis showed that both Blastocystis homologues grouped with those of the archaea into a strongly supported clade, indicating lateral acquisition of the gene from Methanomicrobiales (Tsaousis et al., 2012). The fused gene is found in the genomes of all Blastocystis subtypes, in addition to the genome of Proteromonas lacertae (found in BioProject: PRJNA386230), a Stramenopile species closely related to Blastocystis. Functional characterization of the Blastocystis protein showed that it binds [4Fe-4S] clusters and has ATPase activity. The protein was shown to localize in the cytosol of the parasite and to be overexpressed under oxygen-stressed conditions (Tsaousis et al., 2012). This was unsurprising, since in various bacteria, it has been demonstrated that the machinery is overexpressed under oxygen stress or iron depletion conditions, in order to support the potentially damaged apo-proteins of the cell (Rangachari et al., 2002; Mettert et al., 2008).
Following its discovery in Blastocystis, a fused SufCB gene was later found in other distantly related microbial eukaryotes. The first was the breviate Pygsuia biforma, a free-living anaerobe, but aerotolerant amoeboid flagellate isolated from hypoxic marine sediments. The organism branches at the base of the eukaryotic supergroup Obazoa, which is comprised of animals, fungi, and apusomonads (Figure 3B). The P. biforma genome encodes two homologues of the protein (Stairs et al., 2014). Localization experiments showed that one homologue localizes in mitochondria, while the other localizes in the cytosol (Stairs et al., 2014). Phylogenetic analysis showed that both $P$. biforma homologues branch closely with those of Blastocystis. Interestingly, analysis of the RNA-seq data did not show expression of any of the components of the mitochondrial ISC machinery, while components of the CIA machinery (Cia1, Nbp35, Cfd1, Nar1, Cia2, and Met18) were present (Stairs et al., 2014).

A fused SufCB gene was also found in Stygiella incarcerata along with genes encoding components of the mitochondrial ISC machinery (Leger et al., 2016). Stygiella incarcerata a microaerophilic jakobid flagellate inhabiting anoxic environments and is distantly related to Stramenopiles and Breviata (e.g., Blastocystis and Pygsuia, respectively; Figure 3B). The SUFCB gene of $S$. incarcerata displayed the same characteristics as the homologues of Blastocystis and Pygsuia, and it lacked mitochondrial targeting peptides suggesting a potential cytosolic localization. While the authors did not find any introns in the transcriptome derived fused gene, data from the closely related jakobid Velundella trypanoides (found in BioProject: PRJNA268717) also demonstrated the presence of a homologue (Leger et al., 2016), suggesting that the gene is likely not a contaminant. Phylogenetic analysis showed that the SUF eukaryotic homologues from Blastocystis, Pygsuia, and Stygiella formed a strongly supported clade, with Methanomicrobiales as a well-supported sister group (Leger et al., 2016), consistent with previous observations (Tsaousis et al., 2012; Stairs et al., 2014). How is it possible for organisms that are so distantly related to have a SUFCB homologue?

Various scenarios could explain the presence of this machinery in at least three eukaryotic lineages. Herein, I will discuss three scenarios (Figures 3C-G) while providing pros and cons for each hypothesis:

\section{First Theory}

All three organisms (or their ancestors) acquired the methanoarchaeal SufCB independently, likely while inhabiting the same environmental niche (Figure $3 \mathrm{C}$ ). This scenario suggests three independent transfers: once in the common ancestor of Blastocystis and Proteromonas, once in Stygiella, and once in Pygsuia. Each transfer would require co-existence of the donor lineage with each eukaryote separately. Consequently, this setting implies that the ancestors of these organisms co-habituated in similar environments with Methanomicrobiales, which allowed for transfer and incorporation of genes in their genomes. The intriguing question, under this scenario, is why only a single fused gene was transferred or incorporated from these methanomicrobes in the genomes of diverse protozoa lineages (Tsaousis et al., 2012)? 


\section{Second Theory}

The methanoarchaeal SufCB gene was acquired by one of the three eukaryotic organisms (or their ancestors) and then laterally transferred to the others (Figures 3D-F). It is well established that lateral gene transfer events from eukaryotes to eukaryotes are not as uncommon as it was once thought (Danchin, 2016; Eme et al., 2017; Leger et al., 2018). This type of scenario requires that at least two of the protists co-habited with the donor lineage in the same or similar niches at some point of their life cycles. For example, Blastocystis and Proteromonas spend the majority of their life cycle in the gut of various organisms. Nonetheless, Blastocystis is excreted in the environment as a cyst. If cysts were shed in hypoxic environments, then the possibility of Pygsuia and Stygiella encountering Blastocystis (or its ancestor) and subsequently exchanging genetic material is not entirely far-fetched. Interestingly, with the exception of the SufCB gene, to our knowledge, no other genes share the same origins (or clustering) in these three groups.

\section{Third Theory}

The methanoarchaeal SufCB was present in the last eukaryotic common ancestor (LECA) (Figure 3G). The LECA had to have a machinery for the assembly of Fe-S clusters to support its apo-proteins, even before the acquisition of the alphaproteobacterium that gave rise to the present-day mitochondria. Notably, it has been suggested that the CIA machinery, which is present in all eukaryotes investigated so far is a eukaryotic innovation (Tsaousis et al., 2014; Freibert et al., 2017). Since the ISC machinery is found only in mitochondria and the NIF machinery is only present in two closely related organisms, it is unlikely that either one was present in LECA. Thus, an ancestral SUF machinery, which is commonly found in archaea (Outten, 2015), could have been present in LECA. Considering that $\operatorname{SufCB}$ is not only the most "ancient machinery" (Tokumoto et al., 2004) among all biosynthetic apparatuses, but also the most widespread across lineages, it is plausible that the SufCB was present in the common ancestor of eukaryotes as well. The machinery could have either been acquired by a methanoarchaeon or it could have been present in the archaeal group that gave rise to modern eukaryotes (Spang and Ettema, 2017; Zaremba-Niedzwiedzka et al., 2017; Eme et al., 2018). This scenario could explain the presence of a biosynthetic machinery in three distantly related eukaryotic lineages, but it also infers multiple losses of this machinery in the rest of the lineages. Under this scenario, the case of oxymonads is of interest (Karnkowska et al., 2016; Vacek et al., 2018). How can a separate origin of SUF be explained? One explanation would be that the ancestrally acquired SUF was lost and a SUF of different origin was acquired upon loss of mitochondria. Thus, I hypothesize that eukaryotes maintain the chassis that would allow reacquisition of SUF-like machinery. This hypothesis could be tested by incorporating the eukaryotic SUF machineries in various model organisms across the eukaryotic tree of life (e.g., Saccharomyces, Trypanosoma, Tetrahymena, and Dictyostelium). It is worth mentioning that the third theory does not necessary exclude the other theories above.

\section{DISCUSSION: IRON/SULFUR CLUSTER BIOSYNTHESIS DURING THE EVOLUTIONARY HISTORY OF EUKARYOTES}

Given the discovery of this fused gene in diverse lineages of eukaryotes, speculative scenarios propose an initial transfer of the SufCB from an archaeal source into an ancestral microbial eukaryote (Figures 3C,G) and/or lateral gene transfer events to other eukaryotes (Tsaousis et al., 2014; Leger et al., 2016) (Figures 3D-F). Nevertheless, it is imperative to highlight the importance of this pathway in the evolution and adaptation of eukaryotes.

The last eukaryotic common ancestor (LECA) lived about 1.8 billion years ago (Betts et al., 2018) and seems to have been more complicated than was previously thought (Koonin, 2015). It has been speculated that LECA contained organelles and functions that even mirror some of the current microbial eukaryotes, based on comparative genomic analyses with the closest archaeal-relative lineage, the Lokiarchaeota (Spang et al., 2015, 2017, 2018; Zaremba-Niedzwiedzka et al., 2017; Eme et al., 2018; Eme and Ettema, 2018). Among those, it is currently suggested that LECA possessed mitochondria, endomembrane system along with nucleus, actin cytoskeleton, endocytosis and/ or phagocytosis, and a ubiquitin network (Embley and Williams, 2015; Koonin, 2015; Spang et al., 2015; Akil and Robinson, 2018; Eme and Ettema, 2018). Metabolically, based on investigations in Lokiarchaeota, LECA could have been transitioning from anaerobic to aerobic metabolism (due to the acquisition of the mitochondria; aerobic respiration) with a potentially hydrogen-dependent autotrophic lifestyle (Martin et al., 2016; Sousa et al., 2016). Some of these pathways need enzymes (apo-proteins) that require $\mathrm{Fe} / \mathrm{S}$ clusters in order to function, including DNA/RNA polymerases and anaerobic proteins (e.g., pyruvate ferredoxin oxidoreductase; PFO), which have been identified in Lokiarchaeota (Sousa et al., 2016). LECA must have harbored a biosynthetic pathway to support the assembly and trafficking of these $\mathrm{Fe} / \mathrm{S}$ clusters. The presence of a SUF-like machinery in LECA is plausible, since it is the most common machinery amongst archaeal lineages and is also not compartmentalized in most eukaryotes (Tsaousis et al., 2012; Stairs et al., 2014; Karnkowska et al., 2016; Leger et al., 2016). Footprints of this ancient machinery still remain in modern eukaryotes and it is not an invalid prediction that more organisms having this machinery will be discovered. Whether the machineries that are present in Blastocystis/ Proteromonas, Pygsuia, and Stygiella lineages are remnants of the initial machinery (LECA) or later acquisitions (see scenarios Figures 3C-G) will need further investigations; current data clearly illustrate that the CIA and SUF-like machineries can clearly co-exist (Tsaousis et al., 2012, 2014; Stairs et al., 2014; Karnkowska et al., 2016; Leger et al., 2016; Vacek et al., 2018).

It is also important to note that SUF-like machineries have been shown to be upregulated under oxygen stress conditions to support the potential degradation of Fe-S clusters of proteins (Rangachari et al., 2002; Mettert et al., 2008). This function/ support would have been essential during the transformation 
of proto-eukaryotic cells to LECA, since during that period there would have been a transition to increasing concentrations of oxygen (Lane and Martin, 2016). A SUF-like machinery would have been able to compensate for the potential damage of $\mathrm{Fe} / \mathrm{S}$ clusters from oxygen allowing cells to slowly adjust to their new environments. In parallel, acquisition of mitochondria provided not only an oxygen protective compartment for the formation of $\mathrm{Fe} / \mathrm{S}$ clusters, but also the ISC machinery as well (Lill et al., 1999, 2015). Later on, adaptation of these cells to oxygen rich environments and expansion of the CIA machinery in the cytosol along with its ability to "communicate" with the mitochondrial ISC machinery (e.g., ATM1 for transfer of X-factor; Figures 1, 2), resulted into the SUF-like machinery becoming redundant to the ancestors of most eukaryotic lineages. Eukaryotes that still remained under oxygen depleted conditions either retained the SUF-like machinery (scenario Figure 3G) or later acquired a homologue of this (Vacek et al., 2018).

Here, I propose various scenarios on the evolution of the Fe-S cluster machineries in eukaryotes, and I suggest that a SUF-like ancient $\mathrm{Fe} / \mathrm{S}$ cluster machinery could have been present in the proto-eukaryotic cell or LECA. Current omics data do not provide an answer to this question, but existing efforts

\section{REFERENCES}

Akil, C., and Robinson, R. C. (2018). Genomes of Asgard archaea encode profilins that regulate actin. Nature 562, 439-443. doi: 10.1038/ s41586-018-0548-6

Ali, V., and Nozaki, T. (2013). Iron-sulfur clusters, their biosynthesis, and biological functions in protozoan parasites. Adv. Parasitol. 83, 1-92. doi: 10.1016/B978-0-12-407705-8.00001-X

Ali, V., Shigeta, Y., Tokumoto, U., Takahashi, Y., and Nozaki, T. (2004). An intestinal parasitic protist, Entamoeba histolytica, possesses a nonredundant nitrogen fixation-like system for iron-sulfur cluster assembly under anaerobic conditions. J. Biol. Chem. 279, 16863-16874. doi: 10.1074/ jbc.M313314200

Balk, J., and Pilon, M. (2011). Ancient and essential: the assembly of ironsulfur clusters in plants. Trends Plant Sci. 16, 218-226. doi: 10.1016/j. tplants.2010.12.006

Betts, H. C., Puttick, M. N., Clark, J. W., Williams, T. A., Donoghue, P. C. J., and Pisani, D. (2018). Integrated genomic and fossil evidence illuminates life's early evolution and eukaryote origin. Nat. Ecol. Evol. 2, 1556-1562. doi: 10.1038/s41559-018-0644-x

Braymer, J. J., and Lill, R. (2017). Iron-sulfur cluster biogenesis and trafficking in mitochondria. J. Biol. Chem. 292, 12754-12763. doi: 10.1074/jbc.R117.787101

Burki, F., Kaplan, M., Tikhonenkov, D. V., Zlatogursky, V., Minh, B. Q., Radaykina, L. V., et al. (2016). Untangling the early diversification of eukaryotes: a phylogenomic study of the evolutionary origins of Centrohelida, Haptophyta and Cryptista. Proc. R Soc. B Biol. Sci. 283:20152802. doi: 10.1098/ rspb.2015.2802

Couturier, J., Touraine, B., Briat, J. F., Gaymard, F., and Rouhier, N. (2013). The iron-sulfur cluster assembly machineries in plants: current knowledge and open questions. Front. Plant Sci. 4:259. doi: 10.3389/fpls.2013.00259

Danchin, E. G. (2016). Lateral gene transfer in eukaryotes: tip of the iceberg or of the ice cube? BMC Biol. 14:101. doi: 10.1186/s12915-016-0330-x

Embley, T. M., and Martin, W. (2006). Eukaryotic evolution, changes and challenges. Nature 440, 623-630. doi: 10.1038/nature04546

Embley, T. M., and Williams, T. A. (2015). Evolution: steps on the road to eukaryotes. Nature 521, 169-170. doi: 10.1038/nature14522

Eme, L., and Ettema, T. J. G. (2018). The eukaryotic ancestor shapes up. Nature 562, 352-353. doi: 10.1038/d41586-018-06868-2 to broadly sample the large diversity of archaeal and eukaryotic lineages could provide the missing pieces of this unsolved puzzle.

\section{AUTHOR CONTRIBUTIONS}

The author confirms being the sole contributor of this work and has approved it for publication.

\section{FUNDING}

AT was supported by an internal grant from the University of Kent and a Biotechnology and Biological Sciences Research Council research grant (BB/M009971/1).

\section{ACKNOWLEDGMENTS}

I would like to thank Dr. Eleni Gentekaki for her constructive comments and critique on the manuscript. I would also like to thank Dr. Joel Dacks for providing me the phylogenetic tree showing the relationship between eukaryotes that was used in Figure 3.

Eme, L., Gentekaki, E., Curtis, B., Archibald, J. M., and Roger, A. J. (2017). Lateral gene transfer in the adaptation of the anaerobic parasite blastocystis to the gut. Curr. Biol. 27, 807-820. doi: 10.1016/j.cub.2017.02.003

Eme, L., Spang, A., Lombard, J., Stairs, C. W., and Ettema, T. J. G. (2018). Archaea and the origin of eukaryotes. Nat. Rev. Microbiol. 16, 120. doi: 10.1038/nrmicro.2017.154

Fontecave, M., Choudens, S. O., Py, B., and Barras, F. (2005). Mechanisms of iron-sulfur cluster assembly: the SUF machinery. J. Biol. Inorg. Chem. 10, 713-721. doi: 10.1007/s00775-005-0025-1

Freibert, S. A., Goldberg, A. V., Hacker, C., Molik, S., Dean, P., Williams, T. A., et al. (2017). Evolutionary conservation and in vitro reconstitution of microsporidian iron-sulfur cluster biosynthesis. Nat. Commun. 8:13932. doi: $10.1038 /$ ncomms 13932

Gabaldon, T. (2018). Relative timing of mitochondrial endosymbiosis and the "pre-mitochondrial symbioses" hypothesis. IUBMB Life 70, 1188-1196. doi: 10.1002/iub. 1950

Gawryluk, R. M. R. (2018). Evolutionary biology: a new home for the powerhouse? Curr. Biol. 28, R798-R800. doi: 10.1016/j.cub.2018.05.073

Goldberg, A. V., Molik, S., Tsaousis, A. D., Neumann, K., Kuhnke, G., Delbac, F., et al. (2008). Localization and functionality of microsporidian iron-sulfur cluster assembly proteins. Nature 452, 624-628. doi: 10.1038/ nature06606

Gray, M. W., Burger, G., and Lang, B. F. (1999). Mitochondrial evolution. Science 283, 1476-1481. doi: 10.1126/science.283.5407.1476

Gray, M. W., Burger, G., and Lang, B. F. (2001). The origin and early evolution of mitochondria. Genome Biol. 2:REVIEWS1018. doi: 10.1186/gb-2001-2-6reviews 1018

Grosche, C., Diehl, A., Rensing, S. A., and Maier, U. G. (2018). Iron-sulfur cluster biosynthesis in algae with complex plastids. Genome Biol. Evol. 10, 2061-2071. doi: 10.1093/gbe/evy156

Hirabayashi, K., Yuda, E., Tanaka, N., Katayama, S., Iwasaki, K., Matsumoto, T. et al. (2015). Functional dynamics revealed by the structure of the SufBCD complex, a novel ATP-binding cassette $(\mathrm{ABC})$ protein that serves as a scaffold for iron-sulfur cluster biogenesis. J. Biol. Chem. 290, 29717-29731. doi: 10.1074/jbc.M115.680934

Hjort, K., Goldberg, A. V., Tsaousis, A. D., Hirt, R. P., and Embley, T. M. (2010). Diversity and reductive evolution of mitochondria among microbial eukaryotes. Philos. Trans. R. Soc. Lond. Ser. B Biol. Sci. 365, 713-727. doi: 10.1098/rstb.2009.0224 
Karnkowska, A., Vacek, V., Zubacova, Z., Treitli, S. C., Petrzelkova, R., Eme, L., et al. (2016). A eukaryote without a mitochondrial organelle. Curr. Biol. 26, 1274-1284. doi: 10.1016/j.cub.2016.03.053

Koonin, E. V. (2015). Origin of eukaryotes from within archaea, archaeal eukaryome and bursts of gene gain: eukaryogenesis just made easier? Philos. Trans. R. Soc. Lond. Ser. B Biol. Sci. 370:20140333. doi: 10.1098/rstb.2014.0333

Lane, N., and Martin, W. F. (2015). Eukaryotes really are special, and mitochondria are why. Proc. Natl. Acad. Sci. USA 112:E4823. doi: 10.1073/pnas.1509237112

Lane, N., and Martin, W. F. (2016). Mitochondria, complexity, and evolutionary deficit spending. Proc. Natl. Acad. Sci. USA 113:E666. doi: 10.1073/ pnas. 1522213113

Le Corguille, G., Pearson, G., Valente, M., Viegas, C., Gschloessl, B., Corre, E., et al. (2009). Plastid genomes of two brown algae, Ectocarpus siliculosus and Fucus vesiculosus: further insights on the evolution of red-algal derived plastids. BMC Evol. Biol. 9:253. doi: 10.1186/1471-2148-9-253

Leger, M. M., Eme, L., Hug, L. A., and Roger, A. J. (2016). Novel hydrogenosomes in the microaerophilic jakobid stygiella incarcerata. Mol. Biol. Evol. 33, 2318-2336. doi: 10.1093/molbev/msw103

Leger, M. M., Eme, L., Stairs, C. W., and Roger, A. J. (2018). Demystifying eukaryote lateral gene transfer (response to Martin 2017 doi: 10.1002/ bies.201700115). BioEssays 40:e1700242. doi: 10.1002/bies.201700242

Lill, R. (2009). Function and biogenesis of iron-sulfur proteins. Nature 460, 831-838. doi: 10.1038/nature08301

Lill, R., Diekert, K., Kaut, A., Lange, H., Pelzer, W., Prohl, C., et al. (1999). The essential role of mitochondria in the biogenesis of cellular iron-sulfur proteins. Biol. Chem. 380, 1157-1166. doi: 10.1515/BC.1999.147

Lill, R., Dutkiewicz, R., Freibert, S. A., Heidenreich, T., Mascarenhas, J., Netz, D. J., et al. (2015). The role of mitochondria and the CIA machinery in the maturation of cytosolic and nuclear iron-sulfur proteins. Eur. J. Cell Biol. 94, 280-291. doi: 10.1016/j.ejcb.2015.05.002

Lill, R., Hoffmann, B., Molik, S., Pierik, A. J., Rietzschel, N., Stehling, O., et al. (2012). The role of mitochondria in cellular iron-sulfur protein biogenesis and iron metabolism. Biochim. Biophys. Acta 1823, 1491-1508. doi: 10.1016/j. bbamcr.2012.05.009

Maralikova, B., Ali, V., Nakada-Tsukui, K., Nozaki, T., van der Giezen, M., Henze, K., et al. (2010). Bacterial-type oxygen detoxification and iron-sulfur cluster assembly in amoebal relict mitochondria. Cell. Microbiol. 12, 331-342. doi: $10.1111 /$ j.1462-5822.2009.01397.x

Margulis, L., Chapman, M., Guerrero, R., and Hall, J. (2006). The last eukaryotic common ancestor (LECA): acquisition of cytoskeletal motility from aerotolerant spirochetes in the Proterozoic eon. Proc. Natl. Acad. Sci. USA 103, 13080-13085. doi: $10.1073 /$ pnas. 0604985103

Martijn, J., Vosseberg, J., Guy, L., Offre, P., and Ettema, T. J. G. (2018). Deep mitochondrial origin outside the sampled alphaproteobacteria. Nature 557, 101-105. doi: 10.1038/s41586-018-0059-5

Martin, W. F., Neukirchen, S., Zimorski, V., Gould, S. B., and Sousa, F. L. (2016). Energy for two: new archaeal lineages and the origin of mitochondria. BioEssays 38, 850-856. doi: 10.1002/bies.201600089

Mettert, E. L., Outten, F. W., Wanta, B., and Kiley, P. J. (2008). The impact of $\mathrm{O}(2)$ on the $\mathrm{Fe}-\mathrm{S}$ cluster biogenesis requirements of Escherichia coli FNR. J. Mol. Biol. 384, 798-811. doi: 10.1016/j.jmb.2008.09.080

Miller, C. N., Josse, L., and Tsaousis, A. D. (2018). Localization of Fe-S biosynthesis machinery in Cryptosporidium parvum mitosome. J. Eukaryot. Microbiol. 65, 913-922. doi: 10.1111/jeu.12663

Muller, M. (1973). Peroxisomes and hydrogenosomes in protozoa. J. Histochem. Cytochem. 21, 955-957. doi: 10.1177/21.11.955

Nyvltova, E., Sutak, R., Harant, K., Sedinova, M., Hrdy, I., Paces, J., et al. (2013). NIF-type iron-sulfur cluster assembly system is duplicated and distributed in the mitochondria and cytosol of Mastigamoeba balamuthi. Proc. Natl. Acad. Sci. USA 110, 7371-7376. doi: 10.1073/pnas.1219590110

Outten, F. W. (2015). Recent advances in the Suf Fe-S cluster biogenesis pathway: beyond the Proteobacteria. Biochim. Biophys. Acta 1853, 1464-1469. doi: 10.1016/j.bbamcr.2014.11.001

Paul, V. D., Muhlenhoff, U., Stumpfig, M., Seebacher, J., Kugler, K. G., Renicke, C., et al. (2015). The deca-GX3 proteins Yae1-Lto1 function as adaptors recruiting the $\mathrm{ABC}$ protein Rli1 for iron-sulfur cluster insertion. eLife 4:e08231. doi: 10.7554/eLife. 08231

Pena-Diaz, P., and Lukes, J. (2018). Fe-S cluster assembly in the supergroup Excavata. J. Biol. Inorg. Chem. 23, 521-541. doi: 10.1007/s00775-018-1556-6
Pittis, A. A., and Gabaldon, T. (2016). Late acquisition of mitochondria by a host with chimaeric prokaryotic ancestry. Nature 531, 101-104. doi: 10.1038/ nature16941

Pyrih, J., Pyrihova, E., Kolisko, M., Stojanovova, D., Basu, S., Harant, K., et al. (2016). Minimal cytosolic iron-sulfur cluster assembly machinery of Giardia intestinalis is partially associated with mitosomes. Mol. Microbiol. 102, 701-714. doi: 10.1111/mmi.13487

Rangachari, K., Davis, C. T., Eccleston, J. F., Hirst, E. M., Saldanha, J. W., Strath, M., et al. (2002). SufC hydrolyzes ATP and interacts with SufB from Thermotoga maritima. FEBS Lett. 514, 225-228. doi: 10.1016/S0014-5793(02)02369-4

Rouault, T. A., and Maio, N. (2017). Biogenesis and functions of mammalian iron-sulfur proteins in the regulation of iron homeostasis and pivotal metabolic pathways. J. Biol. Chem. 292, 12744-12753. doi: 10.1074/jbc.R117.789537

Rudolf, J., Makrantoni, V., Ingledew, W. J., Stark, M. J., and White, M. F. (2006). The DNA repair helicases XPD and FancJ have essential iron-sulfur domains. Mol. Cell 23, 801-808. doi: 10.1016/j.molcel.2006.07.019

Santos, H. J., Makiuchi, T., and Nozaki, T. (2018). Reinventing an organelle: the reduced mitochondrion in parasitic protists. Trends Parasitol. 34, 1038-1055. doi: $10.1016 /$ j.pt.2018.08.008

Sousa, F. L., Neukirchen, S., Allen, J. F., Lane, N., and Martin, W. F. (2016). Lokiarchaeon is hydrogen dependent. Nat. Microbiol. 1:16034. doi: 10.1038/ nmicrobiol.2016.34

Spang, A., Caceres, E. F., and Ettema, T. J. G. (2017). Genomic exploration of the diversity, ecology, and evolution of the archaeal domain of life. Science 357. doi: 10.1126/science.aaf3883

Spang, A., Eme, L., Saw, J. H., Caceres, E. F., Zaremba-Niedzwiedzka, K., Lombard, J., et al. (2018). Asgard archaea are the closest prokaryotic relatives of eukaryotes. PLoS Genet. 14:e1007080. doi: 10.1371/journal.pgen.1007080

Spang, A., and Ettema, T. J. G. (2017). Archaeal evolution: the methanogenic roots of archaea. Nat. Microbiol. 2:17109. doi: 10.1038/nmicrobiol.2017.109

Spang, A., Saw, J. H., Jorgensen, S. L., Zaremba-Niedzwiedzka, K., Martijn, J., Lind, A. E., et al. (2015). Complex archaea that bridge the gap between prokaryotes and eukaryotes. Nature 521, 173-179. doi: 10.1038/nature14447

Stairs, C. W., Eme, L., Brown, M. W., Mutsaers, C., Susko, E., Dellaire, G., et al. (2014). A SUF Fe-S cluster biogenesis system in the mitochondrionrelated organelles of the anaerobic protist Pygsuia. Curr. Biol. 24, 1176-1186. doi: 10.1016/j.cub.2014.04.033

Stehling, O., Mascarenhas, J., Vashisht, A. A., Sheftel, A. D., Niggemeyer, B., Rosser, R., et al. (2013). Human CIA2A-FAM96A and CIA2B-FAM96B integrate iron homeostasis and maturation of different subsets of cytosolic-nuclear iron-sulfur proteins. Cell Metab. 18, 187-198. doi: 10.1016/j.cmet.2013.06.015

Stehling, O., Vashisht, A. A., Mascarenhas, J., Jonsson, Z. O., Sharma, T, Netz, D. J., et al. (2012). MMS19 assembles iron-sulfur proteins required for DNA metabolism and genomic integrity. Science 337, 195-199. doi: $10.1126 /$ science. 1219723

Stehling, O., Wilbrecht, C., and Lill, R. (2014). Mitochondrial iron-sulfur protein biogenesis and human disease. Biochimie 100, 61-77. doi: 10.1016/j. biochi.2014.01.010

Tokumoto, U., Kitamura, S., Fukuyama, K., and Takahashi, Y. (2004). Interchangeability and distinct properties of bacterial Fe-S cluster assembly systems: functional replacement of the isc and suf operons in Escherichia coli with the nifSU-like operon from Helicobacter pylori. J. Biochem. 136, 199-209. doi: 10.1093/jb/mvh104

Tonini, M. L., Pena-Diaz, P., Haindrich, A. C., Basu, S., Kriegova, E., Pierik, A. J., et al. (2018). Branched late-steps of the cytosolic iron-sulfur cluster assembly machinery of Trypanosoma brucei. PLoS Pathog. 14:e1007326. doi: 10.1371/ journal.ppat.1007326

Tovar, J., Fischer, A., and Clark, C. G. (1999). The mitosome, a novel organelle related to mitochondria in the amitochondrial parasite Entamoeba histolytica. Mol. Microbiol. 32, 1013-1021. doi: 10.1046/j.1365-2958.1999.01414.x

Tovar, J., Leon-Avila, G., Sanchez, L. B., Sutak, R., Tachezy, J., van der Giezen, M., et al. (2003). Mitochondrial remnant organelles of giardia function in ironsulfur protein maturation. Nature 426, 172-176. doi: 10.1038/nature01945

Treitli, S. C., Kotyk, M., Yubuki, N., Jirounkova, E., Vlasakova, J., Smejkalova, P., et al. (2018). Molecular and morphological diversity of the oxymonad genera monocercomonoides and Blattamonas gen. nov. Protist 169, 744-783. doi: 10.1016/j.protis.2018.06.005

Tsaousis, A. D., Gentekaki, E., Eme, L., Gaston, D., and Roger, A. J. (2014). Evolution of the cytosolic iron-sulfur cluster assembly machinery in Blastocystis 
species and other microbial eukaryotes. Eukaryot. Cell 13, 143-153. doi: 10.1128/EC.00158-13

Tsaousis, A. D., Kunji, E. R., Goldberg, A. V., Lucocq, J. M., Hirt, R. P., and Embley, T. M. (2008). A novel route for ATP acquisition by the remnant mitochondria of Encephalitozoon cuniculi. Nature 453, 553-556. doi: 10.1038/ nature06903

Tsaousis, A. D., Ollagnier de Choudens, S., Gentekaki, E., Long, S., Gaston, D., Stechmann, A., et al. (2012). Evolution of Fe/S cluster biogenesis in the anaerobic parasite Blastocystis. Proc. Natl. Acad. Sci. USA 109, 10426-10431. doi: 10.1073/pnas.1116067109

Vacek, V., Novak, L. V. F., Treitli, S. C., Taborsky, P., Cepicka, I., Kolisko, M., et al. (2018). Fe-S cluster assembly in oxymonads and related protists. Mol. Biol. Evol. 35, 2712-2718. doi: 10.1093/molbev/msy168

van der Giezen, M., Cox, S., and Tovar, J. (2004). The iron-sulfur cluster assembly genes iscS and iscU of Entamoeba histolytica were acquired by horizontal gene transfer. BMC Evol. Biol. 4:7. doi: 10.1186/1471-2148-4-7

Williams, B. A., Hirt, R. P., Lucocq, J. M., and Embley, T. M. (2002). A mitochondrial remnant in the microsporidian Trachipleistophora hominis. Nature 418, 865-869. doi: 10.1038/nature00949
Wollers, S., Layer, G., Garcia-Serres, R., Signor, L., Clemancey, M., Latour, J. M., et al. (2010). Iron-sulfur (Fe-S) cluster assembly: the SufBCD complex is a new type of Fe-S scaffold with a flavin redox cofactor. J. Biol. Chem. 285, 23331-23341. doi: 10.1074/jbc.M110.127449

Zaremba-Niedzwiedzka, K., Caceres, E. F., Saw, J. H., Backstrom, D., Juzokaite, L., Vancaester, E., et al. (2017). Asgard archaea illuminate the origin of eukaryotic cellular complexity. Nature 541, 353-358. doi: 10.1038/ nature 21031

Conflict of Interest: The author declares that the research was conducted in the absence of any commercial or financial relationships that could be construed as a potential conflict of interest.

Copyright (c) 2019 Tsaousis. This is an open-access article distributed under the terms of the Creative Commons Attribution License (CC BY). The use, distribution or reproduction in other forums is permitted, provided the original author(s) and the copyright owner(s) are credited and that the original publication in this journal is cited, in accordance with accepted academic practice. No use, distribution or reproduction is permitted which does not comply with these terms. 